\title{
A 3D Scanner for Transparent Glass
}

\author{
Gonen Eren ${ }^{1,2, \star}$, Olivier Aubreton ${ }^{1}$, Fabrice Meriaudeau $^{1}$, \\ L.A. Sanchez Secades ${ }^{1}$, David Fofi ${ }^{1}$, A. Teoman Naskali ${ }^{2}$, \\ Frederic Truchetet ${ }^{1}$, and Aytul Ercil ${ }^{2}$ \\ 1 University of Burgundy, Le2i Laboratory CNRS UMR 5158, Esplanade Erasme, \\ 21000, Dijon, France \\ 2 Sabanci University, VPA Laboratory, Orhanli-Tuzla, 34956, Istanbul, Turkey \\ goneneren@su.sabanciuniv.edu
}

\begin{abstract}
Many practical tasks in industry, such as automatic inspection or robot vision, often require the scanning of three-dimensional shapes by use of non-contact techniques. However, few methods have been proposed to measure three-dimensional shapes of transparent objects because of the difficulty of dealing with transparency and specularity of the surface. This paper presents a $3 \mathrm{D}$ scanner for transparent glass objects based on Scanning From Heating (SFH), a new method that makes use of local surface heating and thermal imaging.
\end{abstract}

Keywords: Infrared imaging, Three-dimensional image processing, Carbon dioxide laser, Glass.

\section{Introduction}

Three-dimensional scanning has been widely used for many years for reverse engineering and part inspection. A good review of 3D model acquisition techniques can be found in [1]. However, most of these methods are designed to obtain the shape of opaque surfaces with Lambertian reflectance properties. Scanning of transparent objects cannot be properly achieved using these techniques. Figure 1 illustrates this fact and presents a transparent glass object and its reconstruction by a Minolta VI-910 Non Contact 3D Digitizer. The 3D reconstruction of the object is affected by internal refractions and specular reflections.

Researchers have proposed different approaches to deal with transparent objects. Miyazaki et al. have developed a method to determine the surface orientations of transparent objects based on polarization degrees in visible and infrared wavelengths 23]. Hata et al. have developed a shape from distortion technique to recover the surface shape of a glass object [4. Ben-Ezra and Nayar estimated the parameterized surface shape of transparent objects using structure from

\footnotetext{
* We gratefully acknowledge the help of C. Oden, H. Isik, E. Dogan and G. Ciftci from Vistek A.S., M. Akay, H. Yuksek and H. Yavaslar from Sisecam A.S., E.D. Kunt and K. Cakir from AMS and O. Aygun from Flir Systems Turkey. This project was partially supported by SAN-TEZ(00335.STZ.2008-2) and SPICE(FP6-2004-ACCSSA-2).
} 


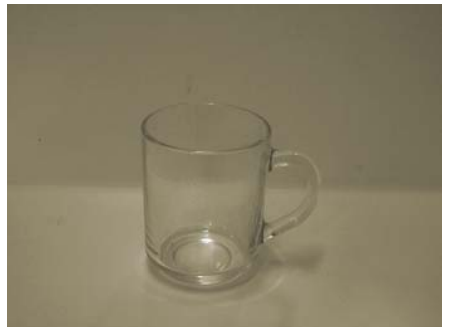

(a)

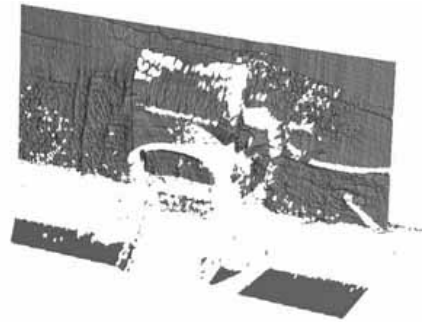

(b)

Fig. 1. (a) Transparent glass object, (b) 3D reconstruction by Minolta VI-910 Non Contact 3D Digitizer

motion [5]. Additionally, an extension using optical flow formulations has been presented by Agarwal et al. [6]. Kutulakos and Steger investigated several applications of direct ray measurement [7]. Fanany et al. proposed a method based on a neural network scheme for transparent surface modeling [8]. Narita et al. used a range-finding approach [9]. Skydan et al. applied a fringe reflection technique to measure the 3D shape of automotive glass [10. These approaches can deal relatively well with different sub-classes of objects. However, the algorithms are still very specific and not generally applicable. Furthermore, many techniques require considerable acquisition effort and careful calibration 11.

In this paper, a new 3D Scanner based on Scanning From Heating (SFH) method is presented. The paper is organized as follows. Section 2 presents the theory behind and describes the scanner. In Section 3, results are presented. Finally, Section 4 concludes the paper.

\section{Scanner Design}

\subsection{Scanning From Heating (SFH) Method}

The working principle of the method is illustrated on Figure 2, An infrared camera and a laser are placed on a moving platform. The surface of the object is localy heated at a point, using the laser. For each position of the moving platform a thermal image is acquired and pixel coordinates of the heated point are calculated $\left(P_{c 1}\left(X_{c 1}, Y_{c 1}\right)\right.$ for Position 1 and $P_{c 2}\left(X_{c 2}, Y_{c 2}\right)$ for Position 2). The pixel coordinates of the heated point are obtained by smoothing the image with a Gaussian filter of $11 \times 11$ pixel size and $\sigma=2.36$, then approximating by a quadratic polynomial in $\mathrm{x}$ and $\mathrm{y}$, and finally examining the polynomial for local maxima. The process is repeated for several points by moving over the object to recover its surface shape. Knowing the intrinsic and extrinsic parameters of the acquisition system, and using the pinhole camera model [12], the 3D world coordinates are obtained as in Eq.(1).

$$
\Delta Z=k \cdot \sqrt{\left(X_{c 2}-X_{c 1}\right)^{2}+\left(Y_{c 2}-Y_{c 1}\right)^{2}}
$$




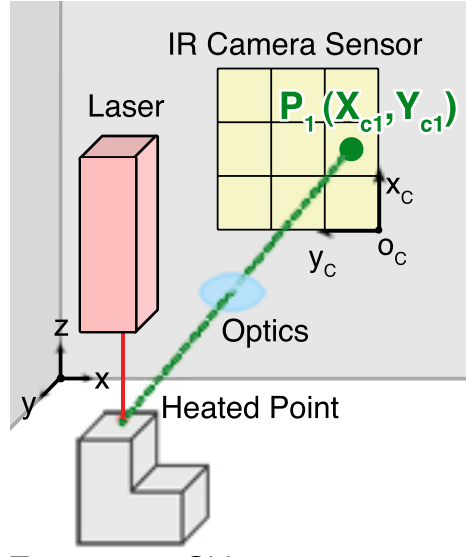

Transparent Object

(a) Position 1

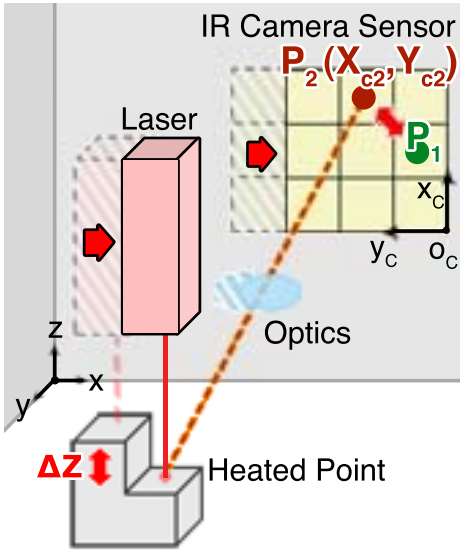

Transparent Object

(b) Position 2

Fig. 2. Scanning From Heating method

where $\Delta Z$ is the variation of the depth between position 1 and $2 . k$ is a constant which can be determined by an initial calibration, which consists of determining the camera coordinates of at least two points of a known object.

The camera observes the emission of the thermal radiation of the heated point once the laser has finished firing. It does not acquire the projection of the laser spot on the surface. Therefore, the method is not affected by the possible reflections of the laser beam on the surface of the glass.

To be able to heat the object with the laser, the object surface should be opaque to the laser source (i.e. the object should not transmit the laser beam through). Figure 3 illustrates the transmission of light as a percentage in the infrared domain of commonly used glasses. Presented glasses are opaque at wavelengths higher than $10 \mu \mathrm{m}$. Consequently, using a laser with a wavelength higher than $10 \mu \mathrm{m}$ as heating source, the SFH method can be applied to transparent glass objects. We chose here to use a $\mathrm{CO}_{2}$ laser at $10.6 \mu \mathrm{m}$.

Once the surface is heated, the thermal camera observes the emission of thermal radiation at the impact point of the laser on the surface. This emission should be omnidirectional so that the thermal camera can capture accurately the heated point on different curvatures of the surface. Figure 4 illustrates emissivity of a dielectric sphere like glass. The emissivity approaches an omnidirectional source.

The prediction of the heat distribution of the laser irradiation zone is important for accurate detection of the heated point. Additionally it is necessary to know the laser power to bring the surface of the object to a given temperature that is detectable by the thermal camera. A mathematical model proposed by Jiao et al. is used [15]. For a $\mathrm{CO}_{2}$ laser beam traveling in direction $x$ at a constant velocity $v$, we consider the laser power to have a Gaussian distribution 


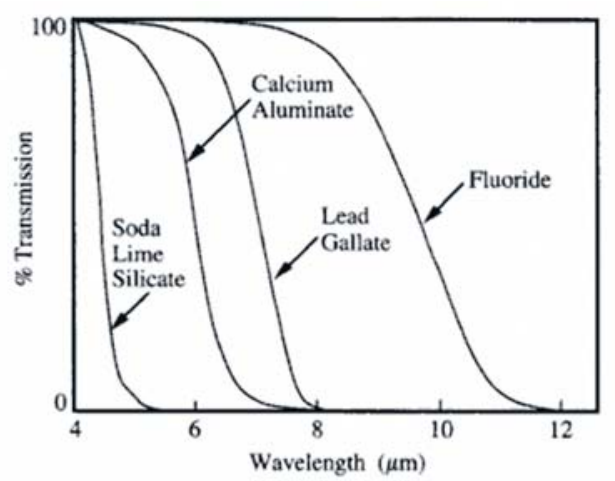

Fig. 3. Transmission of light as a percentage in the infrared domain of commonly used glasses 13 .

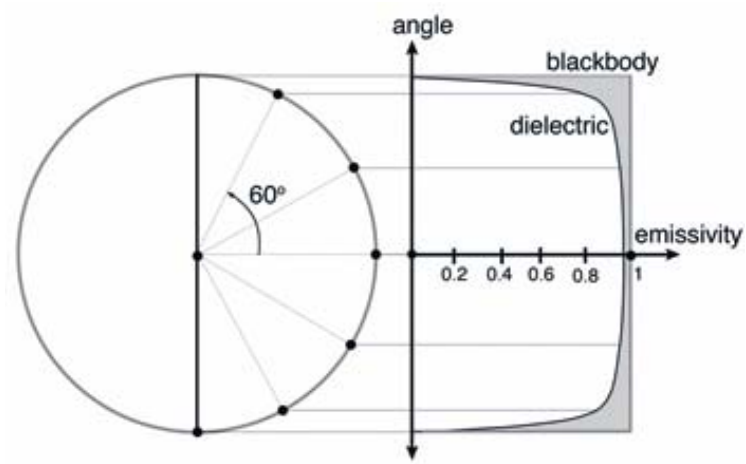

Fig. 4. Emissivity of a dielectric sphere like glass 14

as in Eq. (2). We treat the $\mathrm{CO}_{2}$ laser beam as a surface heating source, so the impulse function $\delta(z)$ is applied in Eq. (2).

$$
I(x, y, z, t)=\frac{P_{0}}{\pi r^{2}} \exp \left(-\frac{(x-v t)^{2}+y^{2}}{r^{2}}\right) \delta(z)
$$

where $P_{0}$ and $r$ are the power and the radius of the $\mathrm{CO}_{2}$ laser beam, respectively.

\subsection{Technical Properties of the Scanner}

The scanner has been designed to reconstruct models of large objects up to $80 \times 150 \times 30 \mathrm{~cm}$ in size. A Synrad 48 Series $10 \mathrm{~W} \mathrm{CO}_{2}$ Laser at $10.6 \mu \mathrm{m}$ is used as the heating source. Thermal images are acquired with a Flir A320G LWIR Camera sensitive to $8-13 \mu \mathrm{m}$. The power of the laser is controlled by a Synrad UC2000 Laser Power Controller. The laser and thermal camera are placed on an $\mathrm{XY}$ positioning system, which is programmable to scan a given area in predefined 


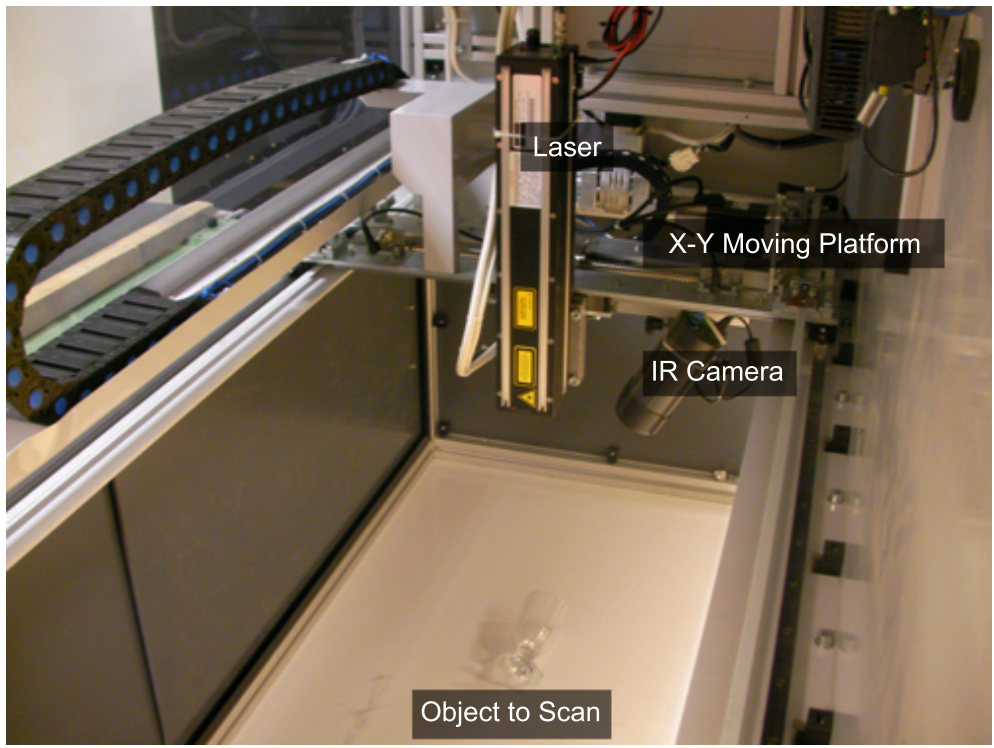

Fig. 5. $\mathrm{CO}_{2}$ Laser, Thermal Camera and the X-Y Moving platform

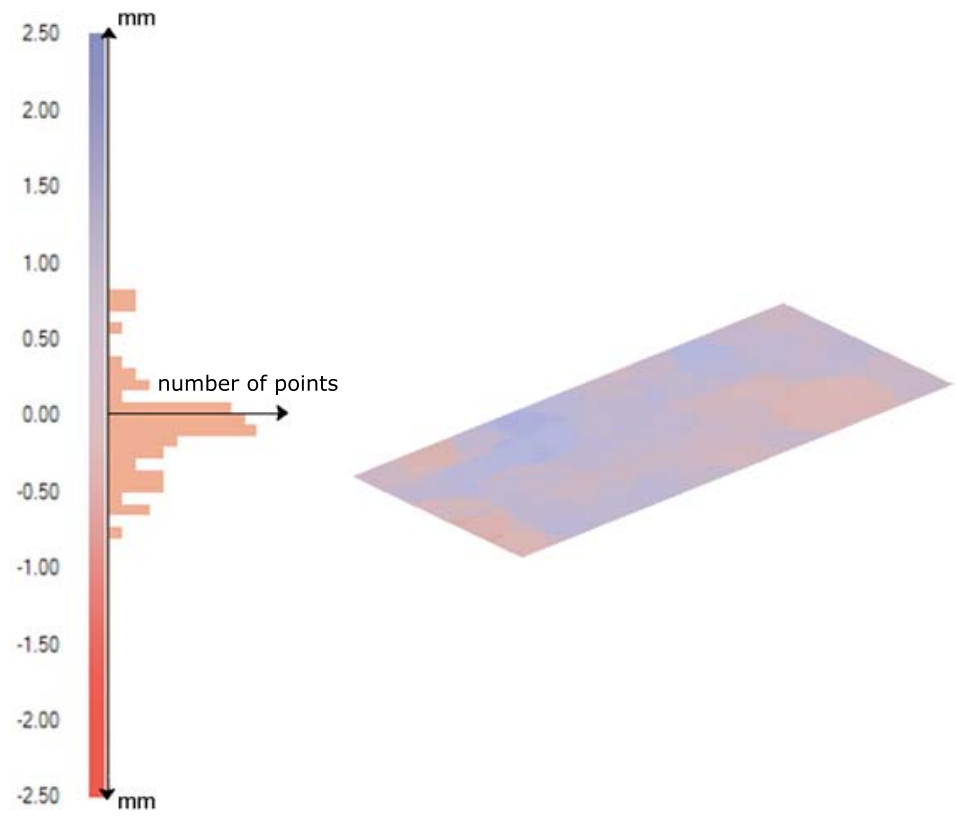

Fig. 6. 3D reconstruction of the transparent glass plate, compared to a perfect plane 
steps of $\mathrm{X}$ and $\mathrm{Y}$ with a precision of $50 \mu \mathrm{m}$ [Fig.5]. Additionally, the laser is set to fire continuously as it moves over the object during the scan, permitting acquisition of thermal images without stopping and the recovery of $3 \mathrm{D}$ points at the maximum speed of the camera (50 fps).

\section{Results}

This section presents results obtained from the SFH scanner.

Figure 6illustrates the results obtained on a glass plate 10x $5 \mathrm{~cm}$ in size from 65 points. The reconstruction is compared to a perfect plane. The average deviation is $150 \mu \mathrm{m}$. This results allows us to validate the method.

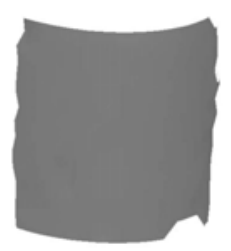

(a)

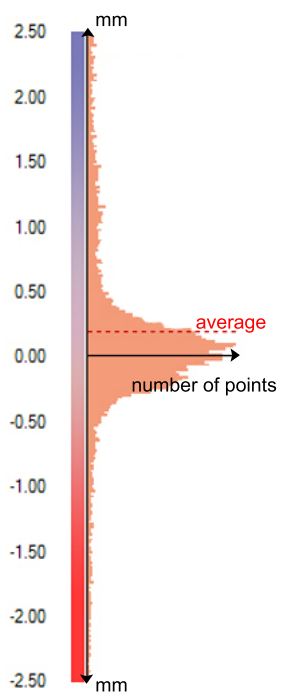

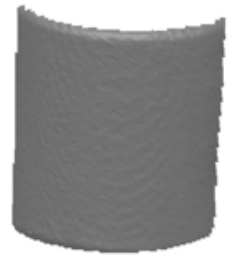

(b)

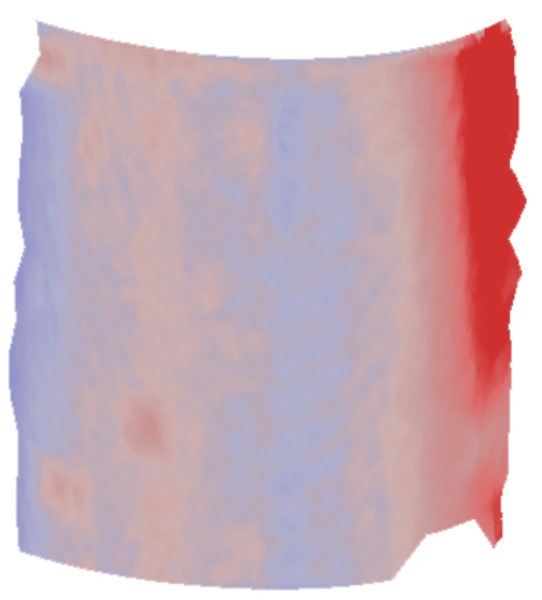

(c)

Fig. 7. (a) 3D reconstruction of the transparent glass cup presented in Fig.1 by the SFH Scanner, (b) 3D reconstruction of the transparent glass cup, after being powdered, by the Minolta 3D Laser Scanner, (c) 3D comparison of the reconstructions and the histogram of the deviation 


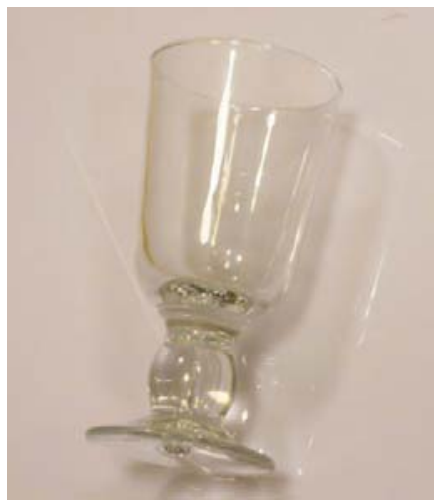

(a)

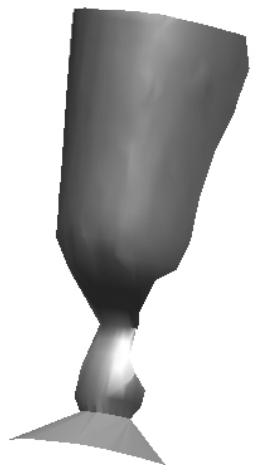

(b)

Fig. 8. (a) Transparent glass object, (b) 3D reconstruction by the SFH Scanner

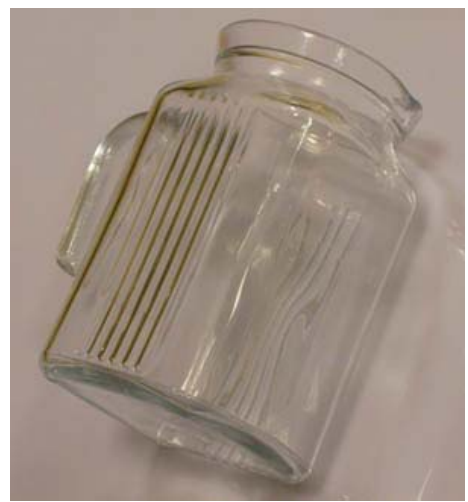

(a)

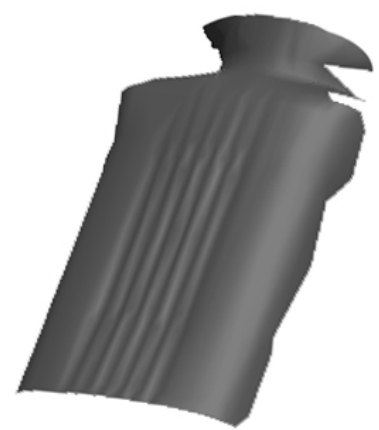

(b)

Fig. 9. (a) Transparent glass object, (b) 3D reconstruction by the SFH Scanner

Figure 7 illustrates the 3D reconstruction of the transparent glass cup presented in Figure 1 by the SFH Scanner and the reconstruction of the same cup, after being powdered, by the Minolta 3D Laser Scanner. In order to validate the efficiency and determine the accuracy of the method, results are compared and the average deviation is determined. Figure 7 presents $3 \mathrm{D}$ comparison of the reconstructions and the histogram of the deviation. The results fit reasonably well, and the average deviation is $210 \mu \mathrm{m}$. Differences between the two models are mostly located on the borders of the scanning region and are probably due to calibration errors in both reconstruction systems.

Figure 8 illustrates the results obtained from the scanning of a complex transparent glass object $7 \times 7 \times 15 \mathrm{~cm}$ in size from 2100 points and Figure 9 illustrates 
the results obtained from another complex transparent glass object 9x9x14cm in size from 1300 points. Hence, the system is applicable to wide range of objects ranging from flat surfaces in the automotive industry to more complex objects in the packaging industry.

\section{Conclusion}

This paper presented a scanner for the 3-D shape measurement of transparent glass objects using the Scanning From Heating method. The scanner was implemented and tested on diverse glass objects. Experiments show that the quality of the reconstructed models is accurate as conventional laser scanners in the visible domain. Additionally, the obtained results demonstrated that the scanner has the capacity to scan different types of surfaces. The scanner holds promise for a wide range of applications in automotive and packaging industries. Future work will concentrate on extensions of the system, mainly oriented toward the projection of a specific pattern (e.g., line, grid, matrix of points) to improve the 3D point acquisition speed and precision. Initial tests on diverse transparent materials such as plastic have been performed and yield promising results. Further, an application for an industrial production line is planned.

\section{References}

1. Bernardini, F., Rushmeier, H.: The 3D Model Acquisition Pipeline. In: Computer Graphics Forum, vol. 21, pp. 149-172. Blackwell Synergy, Malden (2002)

2. Miyazaki, D., Ikeuchi, K.: Inverse Polarization Raytracing: Estimating Surface Shapes of Transparent Objects. In: IEEE Computer Society Conference on Computer Vision and Pattern Recognition, vol. 2, p. 910. Springer, Heidelberg (2005)

3. Miyazaki, D., Saito, M., Sato, Y., Ikeuchi, K.: Determining surface orientations of transparent objects based on polarization degrees in visible and infrared wavelengths. Journal of the Optical Society of America A 19(4), 687-694 (2002)

4. Hata, S., Saitoh, Y., Kumamura, S., Kaida, K.: Shape Extraction of Transparent Object Using Genetic Algorithm. In: International Conference on Pattern Recognition, vol. 13, pp. 684-688 (1996)

5. Ben-Ezra, M., Nayar, S.: What does motion reveal about transparency? In: Proc. IEEE International Conference on Computer Vision, pp. 1025-1032 (2003)

6. Agarwal, S., Mallick, S., Kriegman, D., Belongie, S.: On Refractive Optical Flow. LNCS, pp. 483-494. Springer, Heidelberg (2004)

7. Kutulakos, K., Steger, E.: A Theory of Refractive and Specular 3D Shape by LightPath Triangulation. International Journal of Computer Vision 76(1), 13-29 (2008)

8. Fanany, M., Kumazawa, I., Kobayashi, K.: A neural network scheme for transparent surface modelling. In: Proceedings of the 3rd international conference on Computer graphics and interactive techniques in Australasia and South East Asia, pp. 433-437. ACM, New York (2005)

9. Narita, D., Baba, M., Ohtani, K.: Three-dimensional shape measurement of a transparent object using a rangefinding approach. In: Proceedings of the 20th IEEE Instrumentation and Measurement Technology Conference. IMTC 2003, vol. 2 (2003) 
10. Skydan, O., Lalor, M., Burton, D.: 3D shape measurement of automotive glass by using a fringe reflection technique. Measurement Science and Technology 18(1), 106-114 (2007)

11. Ihrke, I., Kutulakos, K., Lensch, H., Magnor, M., Heidrich, W.: State of the art in transparent and specular object reconstruction. In: STAR Proc. of Eurographics (2008)

12. Forsyth, D., Ponce, J.: Computer Vision: A Modern Approach. Prentice Hall Professional Technical Reference (2002)

13. Shelby, J.: Introduction to Glass Science and Technology. Royal Society of Chemistry (2005)

14. Gaussorgues, G., Chomet, S.: Infrared Thermography. Springer, Heidelberg (1994)

15. Jiao, J., Wang, X.: A numerical simulation of machining glass by dual CO2-laser beams. Optics and Laser Technology 40(2), 297-301 (2008) 